\title{
A DATASET OF BEHAVIOUR OF PEOPLE DURING COVID-19 PANDEMIC IN A WELL DEVELOPING CITY, TAMIL NADU, INDIA
}

\author{
P.Mukesh $^{1}$, AR. Palaniappan ${ }^{2}$, P. Prakashraj ${ }^{3}$ P. Balamurugan ${ }^{4}$ \\ ${ }^{1,2,3,4}$ Department of Civil Engineering, M. Kumarasamy College of Engineering, Karur, Tamilnadu India \\ Email: ${ }^{1}$ mukeshp.civil@mkce.ac.in
}

\begin{abstract}
A dataset of human behaviour during the pandemic situation has been well analysed and reported in this paper. The nature of human behaviour has been dangerous due to high influencing of COVID-19 pandemic situation. This data set clearly describes the expectation of individuals, various communities, and necessity of the lockdown restriction based on the daily new Covid positive cases. It helps to analyse the behavioural response, social, individual expectations and health issues throughout the course of the pandemic period. The result of the present study clearly demonstrates that lockdown restriction highly helps to control the spread of the diseases, and the collective behaviour of humans in the initial period of pandemics depends on basic essential things for their dayto-day life. The dataset revealed that, behaviour of people and their needs has been changed and higher percentage of peoples are willing to go back their business and job to survive in the society against COVID-19.
\end{abstract}

Keywords: Covid-19, Pandemic Situation, Social distance, Human Behaviour

\section{Introduction}

Specifications Table

Subject Needs and behaviour of Human, Diseases Transmittable

Specific subject area Basic Needs, Job willings, Human well being and Disease spread

Type of data Tables, Figures

How data were acquired Data gathered from the Ministry of health and family welfare (Govt. of India)

Data format Raw, Analyzed beahaviour of people

Description of data collection The data were collected during the month of March to June 2020

Data source location Tamilnadu, India

Data accessibility This article contains all the dataset
Value of the Data

The dataset provides information on the daily positive cases, speed of disease spread through community of peoples in Tamil Nadu.

The data are helps to analyze the impact of COVID-19 in urban and rural areas

This dataset is unique to provide the basic needs of peoples and the way to survival of human in the society to against COVID-19

Researchers can utilize the dataset to analyze the behavior of peoples during the pandemic situation

\section{Data description}

Covid-19 is a most dangerous and easily spread virus in the living community. The effects of the virus are very dangerous and reported by [1,2,3]. Tamil Nadu is a one of the most rapid developing state in the way of industrialized, highly densified population in India. It had clocked during the pandemic situation and results that, reducing the economic growth rate of the state. A Tamil Nadu government has answered to the outbreak by subsequent a contact- tracing, testing and surveillance model. More than 85 laboratories have been announced as COVID testing center by Indian council of medical research (ICMR). The first case of the COVID-19 was reported on 7th March 2020. Currently Tamil Nadu has secured the second place in highest positive cases in India [4]. Since 25th March, the first lock down has adhered to more restriction for public transport, industrial activities, vehicle movements and allowed only for medical and basic amenities centers. The restriction and relaxation during the different Lock Down (LD) and Un Lock Down (ULD) period were presented in the Table.1. The LD-1 and LD-2 has followed from 25 th March to 3rd May 2020 and strictly followed to restrict the public transport, vehicle movements and industrial activities. The result of the before lockdown, LD-1 and LD2 shows that, Covid positives cases are recorded as increasing an average rate of 100 cases per day and total numbers of death was 44 (Table.2, 3 and 4). During the LD3 and LD-4 result shows that, average increase in positive 
cases are 84 and 560 respectively and the total death are 18 and 45 respectively (Table.5 and 6). After the month of May, government announced Un Lock down with some restriction such as public transport, government sectors and industries are run with partial number of workers. During the ULD-1 and 2, the Covid cases are the registered average of 1750 and 4500 cases, the total death was recorded as 981 and 2697 respectively. From the dataset, the higher number of deaths was recorded during the ULD-1 and ULD-2.

Table.1 Covid-19 pandemic lockdown schedule in Tamil Nadu, India

\begin{tabular}{|c|c|c|c|c|c|}
\hline Stages & Period & No. ofdays & $\begin{array}{l}\text { Public } \\
\text { Transport }\end{array}$ & $\begin{array}{l}\text { Vehicle } \\
\text { Movement }\end{array}$ & $\begin{array}{l}\text { Industrial } \\
\text { Activities }\end{array}$ \\
\hline LD-1 & $\begin{array}{l}25^{\text {th }} \text { March to } 14^{\text {th }} \\
\text { April } 2020\end{array}$ & 21 & $x$ & $x$ & $x$ \\
\hline LD-2 & $\begin{array}{l}15^{\text {th }} \text { April to } 3^{\text {rd }} \text { May } \\
2020\end{array}$ & 19 & $x$ & $x$ & $x$ \\
\hline LD-3 & $\begin{array}{c}4^{\text {th }} \text { May to } 17^{\text {th }} \text { May } \\
2020\end{array}$ & 14 & $x$ & $\checkmark$ & $\checkmark *$ \\
\hline LD-4 & $\begin{array}{c}18^{\text {th }} \text { May to } 31^{\text {st }} \text { May } \\
2020\end{array}$ & 13 & $x$ & $\checkmark$ & $\checkmark *$ \\
\hline ULD-1 & $\begin{array}{c}1^{\text {st June to } 30^{\text {th }} \text { June }} \\
2020\end{array}$ & 30 & $x$ & $\checkmark$ & $\checkmark^{*}$ \\
\hline ULD-2 & 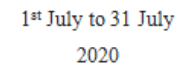 & 31 & $x$ & $\checkmark$ & $\checkmark *$ \\
\hline
\end{tabular}

Table. 2 Covid Positive, recovered death cases during before

\begin{tabular}{cccc}
\multicolumn{2}{c}{ lock down } & \\
\hline Date & Recovered & $\begin{array}{c}\text { De } \\
\text { ath }\end{array}$ & $\begin{array}{c}\text { New } \\
\text { Cases }\end{array}$ \\
\hline $07-03-2020$ & 0 & 0 & 1 \\
$08-03-2020$ & 0 & 0 & 0 \\
$09-03-2020$ & 0 & 0 & 0 \\
$10-03-2020$ & 0 & 0 & 0 \\
$11-03-2020$ & 0 & 0 & 0 \\
$12-03-2020$ & 0 & 0 & 0 \\
$13-03-2020$ & 0 & 0 & 0 \\
$14-03-2020$ & 0 & 0 & 0 \\
$15-03-2020$ & 0 & 0 & 0 \\
$16-03-2020$ & 0 & 0 & 0 \\
$17-03-2020$ & 0 & 0 & 0 \\
$18-03-2020$ & 0 & 0 & 0 \\
$19-03-2020$ & 1 & 0 & 1 \\
$20-03-2020$ & 0 & 0 & 1 \\
$21-03-2020$ & 0 & 0 & 0 \\
$22-03-2020$ & 0 & 0 & 4 \\
$23-03-2020$ & 0 & 0 & 2 \\
$24-03-2020$ & 0 & 0 & 6 \\
\hline
\end{tabular}

Table. 3 Covid Positive, recovered death cases during Lock

\begin{tabular}{cccc}
\multicolumn{2}{c}{ down-1 (LD1) } \\
\hline Date & Recovered & De & New \\
& & ath & Cases \\
\hline $25-03-2020$ & 0 & 0 & 3 \\
$26-03-2020$ & 0 & 1 & 8 \\
$27-03-2020$ & 0 & 0 & 3 \\
$28-03-2020$ & 1 & 0 & 11 \\
$29-03-2020$ & 2 & 0 & 9 \\
$30-03-2020$ & 0 & 0 & 18 \\
$31-03-2020$ & 0 & 0 & 7 \\
$01-04-2020$ & 2 & 0 & 160
\end{tabular}

\begin{tabular}{rlllr}
$02-04-2020$ & & 0 & 0 & 0 \\
$03-04-2020$ & & 0 & & 0 \\
$04-04-2020$ & 0 & 1 & 75 \\
$05-04-2020$ & 0 & 1 & 102 \\
$06-04-2020$ & 2 & 2 & 74 \\
$07-04-2020$ & 0 & 0 & 86 \\
$08-04-2020$ & 11 & 2 & 50 \\
$09-04-2020$ & 2 & 1 & 69 \\
$10-04-2020$ & 0 & 0 & 48 \\
$11-04-2020$ & 23 & 0 & 96 \\
$12-04-2020$ & 0 & 2 & 77 \\
$13-04-2020$ & 6 & 1 & 58 \\
$14-04-2020$ & 8 & 0 & 98 \\
\hline
\end{tabular}

Table.4 Covid Positive, recovered death cases during Lock

\begin{tabular}{|c|c|c|c|}
\hline Date & Recovered & $\begin{array}{c}\text { Dea } \\
\text { th }\end{array}$ & New Cases \\
\hline $15-04-2020$ & 23 & 1 & 31 \\
\hline $16-04-2020$ & 37 & 2 & 38 \\
\hline $17-04-2020$ & 62 & 1 & 25 \\
\hline $18-04-2020$ & 103 & 0 & 56 \\
\hline $19-04-2020$ & 82 & 0 & 49 \\
\hline $20-04-2020$ & 46 & 0 & 105 \\
\hline $21-04-2020$ & 46 & 2 & 43 \\
\hline $22-04-2020$ & 178 & 1 & 76 \\
\hline 23-04-2020 & 27 & 0 & 33 \\
\hline 24-04-2020 & 90 & 2 & 54 \\
\hline $25-04-2020$ & 114 & 2 & 72 \\
\hline 26-04-2020 & 94 & 1 & 66 \\
\hline $27-04-2020$ & 60 & 1 & 64 \\
\hline $28-04-2020$ & 81 & 0 & 52 \\
\hline $29-04-2020$ & 67 & 1 & 121 \\
\hline $30-04-2020$ & 42 & 2 & 104 \\
\hline $01-05-2020$ & 48 & 0 & 161 \\
\hline $02-05-2020$ & 54 & 1 & 203 \\
\hline $03-05-2020$ & 29 & 1 & 231 \\
\hline
\end{tabular}

Table.5 Covid Positive, recovered death cases during Lock

\begin{tabular}{cccc}
\multicolumn{2}{c}{ down-3 (LD3) } & New \\
\hline Date & Recovered & De & Cases \\
& & ath & 266 \\
\hline $04-05-2020$ & 38 & 1 & 527 \\
$05-05-2020$ & 30 & 1 & 508 \\
$06-05-2020$ & 76 & 2 & 771 \\
$07-05-2020$ & 31 & 2 & 580 \\
$08-05-2020$ & 31 & 2 & 600 \\
$09-05-2020$ & 58 & 3 & 526 \\
$10-05-2020$ & 219 & 4 & 669 \\
$11-05-2020$ & 135 & 3 & 798 \\
$12-05-2020$ & 92 & 6 & 716 \\
$13-05-2020$ & 83 & 8 & 509 \\
$14-05-2020$ & 42 & 3 & 447 \\
$15-05-2020$ & 64 & 2 & 434 \\
$16-05-2020$ & 359 & 5 & 477 \\
$17-05-2020$ & 939 & 3 & \\
\hline
\end{tabular}


Table.6 Covid Positive, recovered death cases during Lock

\begin{tabular}{cccc}
\multicolumn{2}{c}{ down-4 (LD4) } & $\begin{array}{c}\text { New } \\
\text { Cases }\end{array}$ \\
\hline Date & $\begin{array}{c}\text { Recovere } \\
\text { d }\end{array}$ & $\begin{array}{c}\text { Dea } \\
\text { th }\end{array}$ & 639 \\
\hline $18-05-2020$ & 634 & 4 & 536 \\
$19-05-2020$ & 234 & 3 & 688 \\
$20-05-2020$ & 489 & 3 & 743 \\
$21-05-2020$ & 987 & 3 & 776 \\
$22-05-2020$ & 400 & 7 & 786 \\
$23-05-2020$ & 846 & 4 & 759 \\
$24-05-2020$ & 363 & 5 & 765 \\
$25-05-2020$ & 833 & 8 & 805 \\
$26-05-2020$ & 407 & 7 & 646 \\
$27-05-2020$ & 611 & 9 & 817 \\
$28-05-2020$ & 567 & 6 & 827 \\
$29-05-2020$ & 639 & 12 & \\
$30-05-2020$ & 765 & 9 &
\end{tabular}

Table.7 Covid Positive, recovered death cases during Unlock down-1 (ULD1)

\begin{tabular}{|c|c|c|c|}
\hline Date & Recovered & $\begin{array}{c}\text { Deat } \\
\mathbf{h}\end{array}$ & New Cases \\
\hline 01-06-2020 & 757 & 13 & 1149 \\
\hline $02-06-2020$ & 413 & 11 & 1162 \\
\hline 03-06-2020 & 536 & 13 & 1091 \\
\hline 04-06-2020 & 610 & 11 & 1286 \\
\hline $05-06-2020$ & 586 & 12 & 1384 \\
\hline $06-06-2020$ & 860 & 12 & 1438 \\
\hline $07-06-2020$ & 633 & 19 & 1458 \\
\hline $08-06-2020$ & 604 & 18 & 1515 \\
\hline 09-06-2020 & 528 & 17 & 1562 \\
\hline $10-06-2020$ & 798 & 21 & 1685 \\
\hline $11-06-2020$ & 1008 & 19 & 1927 \\
\hline $12-06-2020$ & 1372 & 23 & 1875 \\
\hline $13-06-2020$ & 1342 & 18 & 1982 \\
\hline $14-06-2020$ & 1362 & 30 & 1989 \\
\hline $15-06-2020$ & 1138 & 38 & 1974 \\
\hline $16-06-2020$ & 797 & 44 & 1843 \\
\hline $17-06-2020$ & 1438 & 49 & 1515 \\
\hline $18-06-2020$ & 842 & 48 & 2174 \\
\hline $19-06-2020$ & 1017 & 49 & 2141 \\
\hline $20-06-2020$ & 1630 & 41 & 2115 \\
\hline $21-06-2020$ & 1045 & 38 & 2396 \\
\hline $22-06-2020$ & 1438 & 53 & 2532 \\
\hline $23-06-2020$ & 1358 & 37 & 2710 \\
\hline $24-06-2020$ & 1227 & 39 & 2516 \\
\hline $25-06-2020$ & 2424 & 33 & 2865 \\
\hline $26-06-2020$ & 2236 & 45 & 3509 \\
\hline $27-06-2020$ & 1358 & 46 & 3645 \\
\hline $28-06-2020$ & 2737 & 68 & 3713 \\
\hline $29-06-2020$ & 1443 & 54 & 3940 \\
\hline $30-06-2020$ & 2212 & 62 & 3949 \\
\hline
\end{tabular}

Table. 8 Covid Positive, recovered death cases during Unlock down-2 (ULD2)

\begin{tabular}{|c|c|c|c|}
\hline Date & Recovered & $\begin{array}{l}\text { De } \\
\text { ath }\end{array}$ & $\begin{array}{l}\text { New } \\
\text { Cases }\end{array}$ \\
\hline $01-07-2020$ & 2325 & 60 & 3943 \\
\hline $02-07-2020$ & 2852 & 63 & 3882 \\
\hline 03-07-2020 & 3095 & 57 & 4343 \\
\hline 04-07-2020 & 2357 & 64 & 4329 \\
\hline $05-07-2020$ & 2214 & 65 & 4280 \\
\hline $06-07-2020$ & 2186 & 60 & 4150 \\
\hline 07-07-2020 & 3793 & 61 & 3827 \\
\hline 08-07-2020 & 4545 & 65 & 3616 \\
\hline 09-07-2020 & 3051 & 64 & 3756 \\
\hline $10-07-2020$ & 3994 & 65 & 4231 \\
\hline $11-07-2020$ & 4163 & 64 & 3680 \\
\hline $12-07-2020$ & 3591 & 69 & 3965 \\
\hline $13-07-2020$ & 3617 & 68 & 4244 \\
\hline $14-07-2020$ & 3035 & 66 & 4328 \\
\hline $15-07-2020$ & 4743 & 67 & 4526 \\
\hline $16-07-2020$ & 5000 & 68 & 4496 \\
\hline $17-07-2020$ & 5106 & 69 & 4549 \\
\hline $18-07-2020$ & 3391 & 79 & 4538 \\
\hline $19-07-2020$ & 3049 & 88 & 4807 \\
\hline $20-07-2020$ & 4059 & 78 & 4979 \\
\hline $21-07-2020$ & 3861 & 70 & 4985 \\
\hline $22-07-2020$ & 4894 & 75 & 4965 \\
\hline $23-07-2020$ & 4913 & 518 & 5849 \\
\hline $24-07-2020$ & 5210 & 88 & 6472 \\
\hline $25-07-2020$ & 6504 & 88 & 6785 \\
\hline $26-07-2020$ & 7758 & 89 & 6988 \\
\hline $27-07-2020$ & 5471 & 85 & 6986 \\
\hline $28-07-2020$ & 5723 & 77 & 6993 \\
\hline $29-07-2020$ & 4707 & 88 & 6972 \\
\hline $30-07-2020$ & 5927 & 82 & 6426 \\
\hline
\end{tabular}




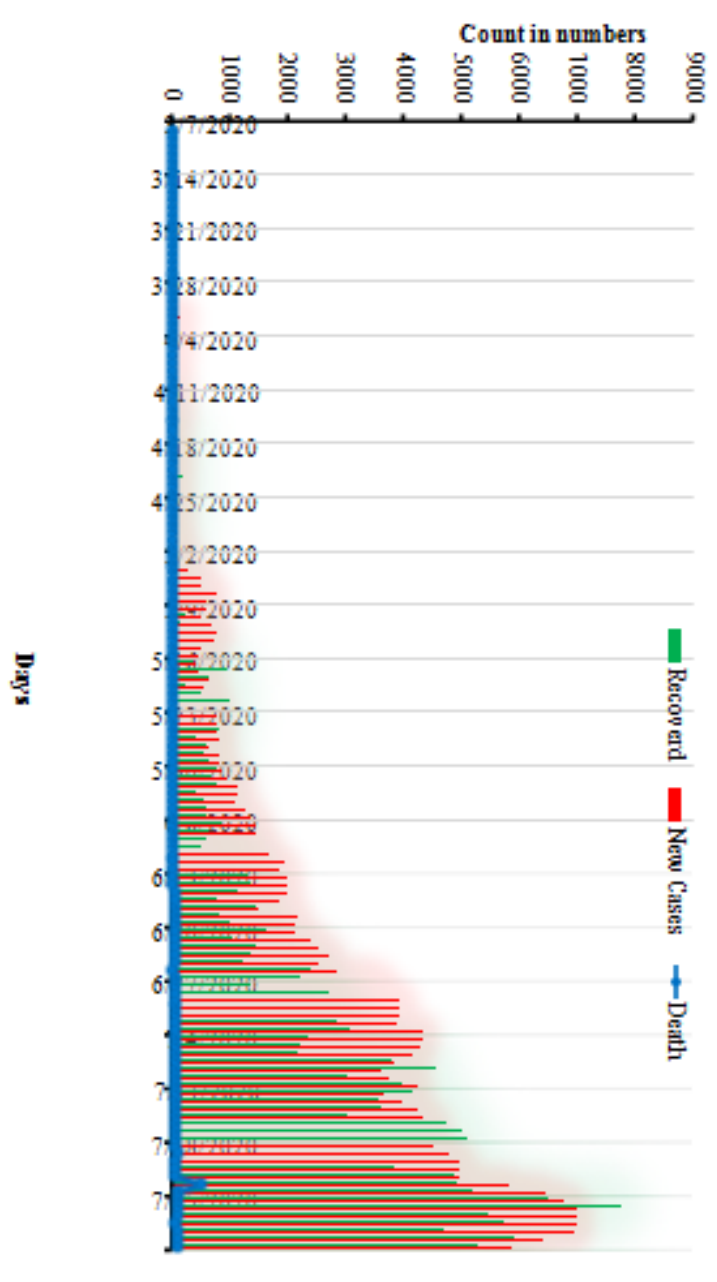

The analysis of the Covid dataset helps to identify the present nature of the peoples and behavior of people living in the pandemic situation [5,6,7]. During the first curfew period (Table 9), $45 \%$ of people said they only wanted to go to the pharmacy. Because at LD1 time people wanted to go out only for essential tasks because of the fear of the Covid19. During this period $25 \%, 10 \%, 10 \%, 4 \%$ and $6 \%$ of the population were interested in going to supermarkets, hospitals, banks, temples, and work, respectively. After the 21 st day of the LD1, people have expressed their interest in going to the supermarket next to the pharmacy to alleviate the shortage of groceries in their homes during the next two and third (LD2 \&amp; LD3) curfews. In the same time the people's interest to go to temples has dropped significantly. During the LD4, ULD1 and ULD2 period 15\%, 25\%, and 19\% of people respectively have expressed interest in going to work for their economic needs. According to Table 10 majority informal workers have expressed a desire to go to work regardless of COVID-19 fears due to their poverty during all covid-19 lockdown periods. It can be seen that their preference is $45 \%$, $42 \%, 45 \%, 39 \%, 45 \%$ and $55 \%$ during the LD1, LD2, LD3, LD4, ULD1, and ULD2 period respectively. This preference decreased during the LD2, LD3 and LD4 as migrant workers migrated to their hometowns. And only 23\%, 23\%, 26\%, $15 \%$ and $15 \%$ of people prefer to work from home during LD2, LD3, LD4, ULD1, and ULD2 curfews except during the LD1 $(43 \%)$, which may have been due to the psychological stress of being at home. And with the exception of the LD1, most formal workers prefer to go to the office during LD2, LD3, LD4, ULD1, and ULD2.

Fig.1 Variation of Covid cases during March to July 2020

\section{Design, Materials and Methods}

Table.9 Basic needs for human during the Pandemic situation

\begin{tabular}{ccccccc}
\hline Phase & Pharmacy & Supermarket & Doctor & Bank & Temple & Job \\
\hline LD-1 & 45 & 25 & 10 & 10 & 4 & 6 \\
LD-2 & 36 & 34 & 15 & 12 & 1 & 2 \\
LD-3 & 32 & 36 & 10 & 10 & 1 & 11 \\
LD-4 & 35 & 26 & 10 & 12 & 2 & 15 \\
ULD-1 & 30 & 20 & 12 & 12 & 1 & 25 \\
ULD-2 & 35 & 25 & 10 & 10 & 1 & 19 \\
\hline
\end{tabular}

\begin{tabular}{|c|c|c|c|}
\hline \multicolumn{3}{|c|}{ Table.10 People willingness for job during the Pandemic situation } \\
\hline Phase & Work from Home & Work onsite & Non-organisationworkers \\
\hline LD-1 & 43 & 12 & 45 \\
\hline LD-2 & 23 & 35 & 42 \\
\hline LD-3 & 23 & 32 & 39 \\
\hline LD-4 & 26 & 45 & 45 \\
\hline ULD-1 & 15 & 35 & 50 \\
\hline ULD-2 & 15 & 35 & 45 \\
\hline
\end{tabular}




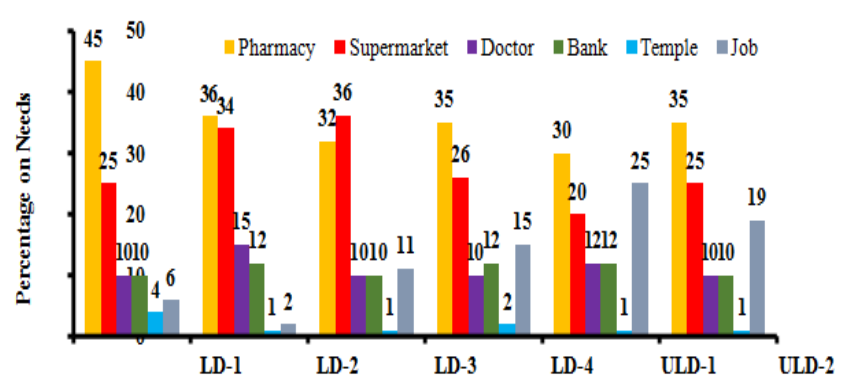

Fig.2 Percentage of people need during the pandemic situation

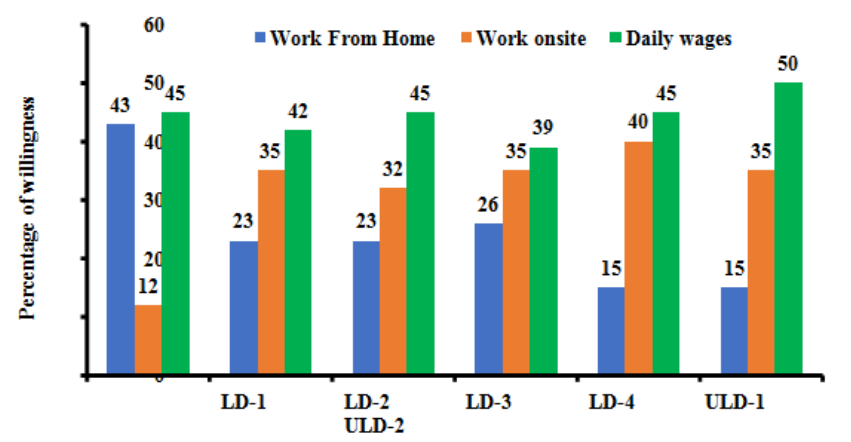

Fig.3 Change in percentage of people willingness during the

\section{References} pandemic situation

[1] Benvenuto, D., Giovanetti, M., Vassallo, L., Angeletti, S., \& Ciccozzi, M. (2020). Application of the ARIMA model on the COVID-2019 epidemic dataset. Data in brief, 105340. https://doi.org/10.1016/j.dib.2020.105340

[2] Morettini, M., Sbrollini, A., Marcantoni, I., \& Burattini, L. (2020). COVID-19 in Italy: Dataset of the Italian Civil Protection Department. Data in Brief, 105526. https://doi.org/10.1016/j.dib.2020.105526

[3] Huynh, T. L. D. (2020). Data for understanding the risk perception of COVID-19 from Vietnamese

[4] Covid related Data collection https://www.mohfw.gov.in/

[5] Machuca-Martinez, F., Amado, R. C., \& Gutierrez, O. (2020). Coronaviruses: A patent dataset report for research and development (R\&D) analysis. Data in Brief, 105551. https://doi.org/10.1016/j.dib.2020.105551

[6] Zanon, D., Manca, A., De Nicolò, A., D'Avolio, A., Musazzi, U. M., Cilurzo, F., \&
[7] Minghetti, P. (2020). Data on the stability of darunavir/cobicistat suspension after tablet manipulation. Data in brief, 105552. https://doi.org/10.1016/j.dib.2020.105552

[8] Trung, T., Hoang, A. D., Nguyen, T. T., Dinh, V. H., Nguyen, Y. C., \& Pham, H. H. (2020). Dataset of Vietnamese student's learning habits during COVID-19. Data in Brief, 105682. https://doi.org/10.1016/j.dib.2020.105682 